\section{The semi-erect position for better visualization of subphrenic hepatocellular carcinoma during ultrasonography examinations}

\author{
Seong Eun Ko', Min Woo Lee ${ }^{1,2}$, Hyo Keun Lim, ${ }^{1,2}$, Ji Hye Min' ${ }^{1}$ Dong Ik Cha', \\ Tae Wook Kang ${ }^{1,2}$, Kyoung Doo Song ${ }^{1,2}$, Min Ju Kim', Hyunchul Rhim ${ }^{1,2}$ \\ ${ }^{1}$ Department of Radiology and Center for Imaging Science, Samsung Medical Center, \\ Sungkyunkwan University School of Medicine, Seoul; ${ }^{2}$ Department of Health Sciences and \\ Technology, SAIHST, Sungkyunkwan University, Seoul, Korea
}

Purpose: This study investigated which body position is more useful for visualizing subphrenic hepatocellular carcinomas (HCCS) during ultrasonography (US) examinations.

Methods: This prospective study was approved by the institutional review board and written informed consent was obtained from all patients. Twenty consecutive patients with a single subphrenic HCC (treatment-naïve, 1 to $3 \mathrm{~cm}$ ) underwent a US examination for planning radiofrequency ablation. The examinations were done by one of three radiologists and the patients were examined in four different body positions - supine, right posterior oblique (RPO), left lateral decubitus (LLD), and semi-erect-by being positioned on a tilted table. The visibility of the index tumor was prospectively assessed using a 4-point scale. Needle insertion was considered to be technically feasible if the visibility score was lower than 2 . The visibility score and technical feasibility were compared using the Wilcoxon signed rank test and the McNemar test, respectively, for pairwise comparisons between different body positions.

Results: The visibility score was significantly lower in the semi-erect position (median, 2; interquartile range, 1 to 2.75$)$ than in the supine (3, 2 to 4$)$, RPO (3, 2 to 4$)$, and $\operatorname{LLD}(4,3.25$ to 4) positions ( $P=0.007, P=0.005$, and $P=0.001$, respectively). The technical feasibility of needle insertion was also significantly higher in the semi-erect position $(75 \%, 15 / 20)$ than in the supine $(45 \%, 9 / 45), \operatorname{RPO}(35 \%, 7 / 20)$, and $\operatorname{LLD}(20 \%, 4 / 20)$ positions $(P=0.031, P=0.021$, and $\mathrm{P}=0.001$, respectively).

Conclusion: The semi-erect position is more useful for the visualization of subphrenic HCCs than the supine, RPO, or LLD positions.

Keywords: Liver; Ultrasound; Surveillance; Position; Hepatocellular carcinoma

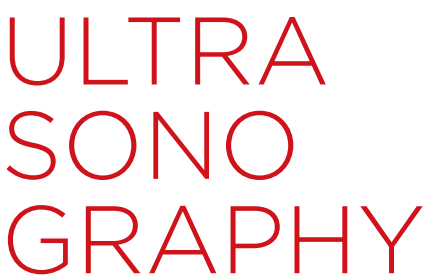

ORIGINAL ARTICLE

https://doi.org/10.14366/usg.20059 pISSN: 2288-5919 • elSSN: 2288-5943 Ultrasonography 2021;40:274-280

Received: April 27, 2020

Revised: June 4, 2020

Accepted: June 22, 2020

Correspondence to:

Min Woo Lee, MD, Department of Radiology and Center for Imaging Science, Samsung Medical Center and Department of Health Sciences and Technology, SAIHST, Sungkyunkwan University, 81 Irwon-ro, Gangnam-gu, Seoul 06351, Korea

Tel. +82-2-3410-2518

Fax. +82-2-3410-2559

E-mail: leeminwoo0@gmail.com

This is an Open Access article distributed under the terms of the Creative Commons Attribution NonCommercial License (http://creativecommons.org/ licenses/by-nc/4.0/) which permits unrestricted noncommercial use distribution, and reproduction in any medium, provided the original work is properly cited.

Copyright @ 2021 Korean Society of Ultrasound in Medicine (KSUM)

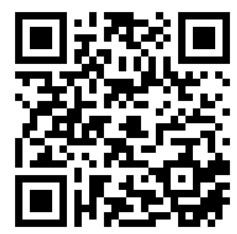

How to cite this article:

Ko SE, Lee MW, Lim HK, Min JH, Cha DI, Kang TW, et al. The semi-erect position for better visualization of subphrenic hepatocellular carcinoma during ultrasonography examinations. Ultrasonography. 2021 Apr;40(2):274-280. 


\section{Introduction}

Ultrasonography (US) is widely used as a surveillance tool for detecting hepatocellular carcinoma (HCC) in patients who are considered to be at high risk for developing HCC $[1,2]$. The goal of a surveillance program is to detect HCC at an early stage when it can be treated either with local therapy or liver transplantation [3]. However, US examinations may be limited by various factors that impact the sensitivity of US for detecting focal hepatic lesions. These include extrinsic factors such as large patient body habitus, a poor sonographic window from overlying lung and rib shadows or bowel gas, and the patient's inability to fully withhold breathing. Furthermore, intrinsic factors such as severe steatosis or fibrosis can impair US beam penetration, which may make the detection of focal hepatic lesions difficult [4].

Tumor location, along with tumor size, is an important factor when detecting small HCCs. For example, subphrenic HCCs are difficult to localize with US even with prior awareness of the tumor location from pre-acquired computed tomography (CT) or magnetic resonance imaging (MRI), which may be explained by US beam attenuation and the poor sonographic window of the subphrenic area $[5,6]$. Consequently, needle insertion aiming for subphrenic HCCS could also be difficult. Although contrast-enhanced US (CEUS) is useful for identifying small HCCs that are inconspicuous on B-mode US, CEUS has a lower sensitivity for deeply positioned tumors such as subphrenic HCCs. In addition, comprehensively assessing the whole liver parenchyma with CEUS may be challenging in the short time window of the arterial phase [7]. Furthermore, a recent study reported that the addition of CEUS to conventional B-mode US did not significantly improve the detection rate of earlystage HCC when used as a surveillance test in a population where hepatitis B virus predominated [8].

To improve visualization of the whole liver during US examinations, patients are examined in various positions, including the supine, left posterior oblique (LPO), right posterior oblique (RPO), and left lateral decubitus (LLD) positions [4]. The semi-erect position may be useful for better visualization of the hepatic dome because in this position, the liver moves down from its original location and the sonographic window is less affected by the lung shadow. Therefore, we postulated that the semi-erect position would be more useful than other positions, including the supine, RPO, and LLD positions, for visualizing subphrenic HCCs. Although position changes are used during US examination of liver in the clinical setting, this topic has not been explored in a scientific manner. Therefore, the purpose of this study was to evaluate which body position was more useful for the visualization of subphrenic HCCs.

\section{Materials and Methods}

\section{Patients}

This prospective study was approved by the institutional review board of our institution, and written informed consent was obtained from all patients. Between June 2013 and May 2014, 20 consecutive patients with subphrenic HCC who met the following inclusion criteria were enrolled in our study: (1) patients with treatment-naïve single HCC (1 to $3 \mathrm{~cm}$ ) detected with contrast-enhanced CT or MRI within 1 month before the planning US examination, (2) patients with tumors in the subphrenic location, defined by the distance between the upper part of the tumor and the diaphragm being less than $1 \mathrm{~cm}$, (3) patients who were referred for planning US for radiofrequency ablation (RFA), (4) patients who were classified as Child-Pugh class A or B, and (5) patients who agreed to participate in this study. The diagnosis of HCC was based on the typical imaging features on CT or MRI, according to the American Association for the Study of Liver Disease guideline [9].

The exclusion criteria were as follows: (1) patients with tumor thrombi on CT or MRI, (2) patients with ascites, (3) patients who previously underwent surgical resection of the liver, and (4) patients over 80 years old.

\section{Planning US in Four Different Positions}

The US examination was performed by one of three radiologists (H.K.L., H.R., and M.W.L.), who had at least 8 years of experience in RFA of HCCs. Before conducting planning US for RFA, the radiologists reviewed pre-acquired $\mathrm{CT}$ or MRI scans and checked for tumor size, tumor location, and the distance from the diaphragm to the index tumor on coronal images of $\mathrm{CT}$ or MRI. The patients were examined in four different body positions-supine, RPO, LLD, and semi-erect-by being positioned on a table (JS-002, Jinsol Medical, Gwangju, Korea) that was capable of tilting. The US examination was first performed in a supine position with fusion imaging (volume navigation, LOGIQ E9, GE Healthcare, Milwaukee, WI, USA) of realtime US and pre-acquired CT/MR images $[10,11]$. Based on the fused CT/MR images, the radiologists localized the index tumor using peritumoral anatomic landmarks such as portal or hepatic vein branches. They also determined whether the index tumor was visible on US and whether the lesion conspicuity was sufficient for needle placement.

The visibility of the index tumor was prospectively assessed at the time of the US examination using the following visibility scores: 1: completely identifiable, highly confident in identifying the index tumor; 2: partially (more than half of the index tumor) identifiable, and confident in identifying the index tumor; 3: partially (less than half of the index tumor) identifiable, but less confident due to poor 
sonic window; and 4: completely unidentifiable. The US examination and visibility score grading were repeated with the patient in the $\operatorname{RPO}\left(30^{\circ}-60^{\circ}\right), \operatorname{LLD}\left(90^{\circ}\right)$, and semi-erect positions $\left(30^{\circ}-40^{\circ}\right)$ (Fig. 1).

\section{Outcome Assessment and Statistical Analysis}

For the baseline characteristics of the patients included in the study, descriptive statistics were presented (number with percentage for categorical data and median with interquartile range [IQR] for continuous data). The visibility scores of the subphrenic HCCS were compared using the Wilcoxon signed rank test for pairwise comparisons between different body positions. Needle insertion was considered to be technically feasible if the visibility score was equal to or lower than 2 . We also compared the technical feasibility of

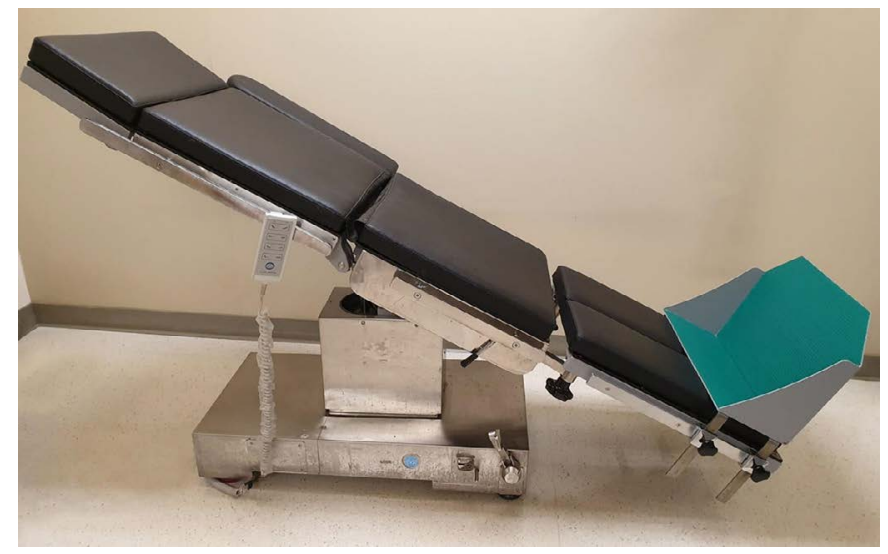

A
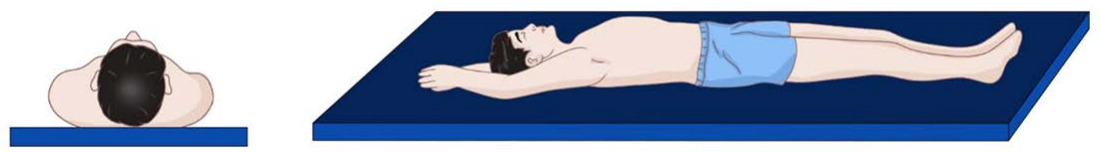

B
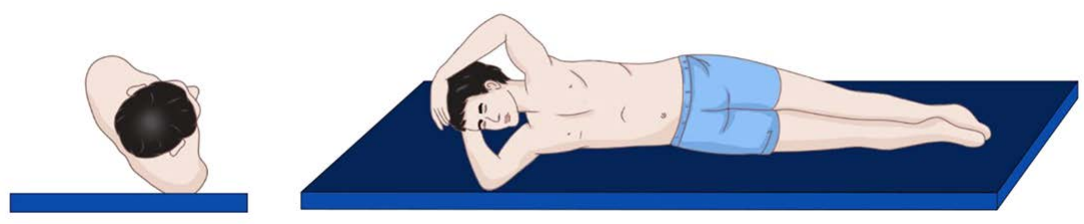

C
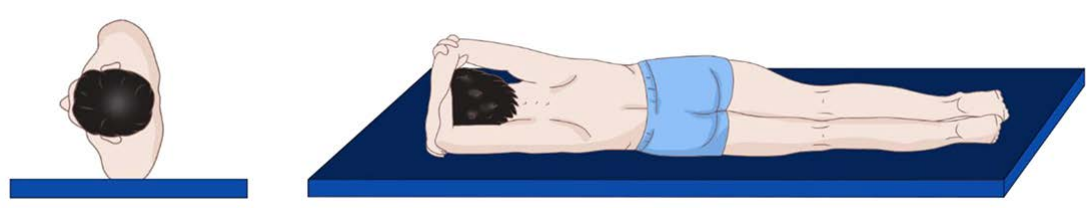

D

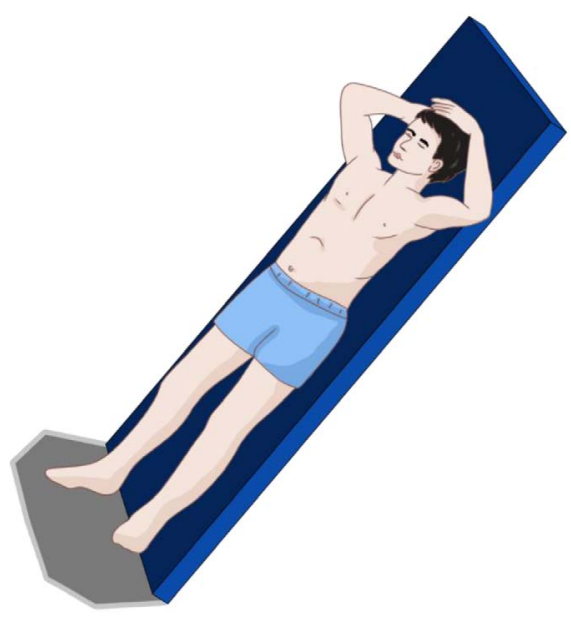

$\mathrm{E}$ 
Table 1. Baseline characteristics of 20 patients

\begin{tabular}{lc}
\hline \multicolumn{1}{c}{ Variable } & Value \\
\hline Age (year) & $62.5(48-74)$ \\
Sex (male/female) & $19 / 1$ \\
Etiology (HBV/HCV/others) & $16(80.0) / 2(10.0) / 2(10.0)$ \\
Child-Pugh class A & $20(100)$ \\
Albumin (g/dL) & $4.4(3.5-5.1)$ \\
Total bilirubin (mg/dL) & $1.1(0.4-1.8)$ \\
PT (INR) & $1.05(0.93-1.31)$ \\
Serum AFP (ng/mL) & $6.2(1.3-272.4)$ \\
PIVKA-II (mAU/mL) & $22.0(16.0-732.0)$ \\
Tumor size (cm) & $1.4(1.1-2.5)$ \\
Distance between diaphragm and lower & $1.5(0.9-3.0)$ \\
margin of the tumor (cm) & \\
Tumor location (Couinaud) & \\
Segment 4 & $2(10.0)$ \\
Segment 7 & $3(15.0)$ \\
Segment 8 & $15(75.0)$ \\
\hline
\end{tabular}

Values are presented as median (range) or number of patients (\%). $\mathrm{HBV}$, hepatitis B virus; $\mathrm{HCV}$, hepatitis C virus; $\mathrm{PT}$, prothrombin time; INR, international normalized ratio; AFP, a-fetoprotein; PIVKA-II, protein induced by vitamin $\mathrm{K}$ absence or antagonist-II.

no difficulty being examined in the four different body positions.

\section{Visibility Scores in Different Body Positions}

The visibility scores are summarized in Fig. 2. In the 20 patients, the median visibility score was 3 (IQR, 2 to 4 ) in the supine position. The median visibility scores were 3 (IQR, 2 to 4 ) in the RPO position, 4 (IQR, 3.25 to 4$)$ in the LLD position, and 2 (IQR, 1 to 2.75$)$ in the semi-erect position. The semi-erect position had the lowest visibility score, with statistically significant differences compared to the supine, RPO, and LLD positions ( $P=0.007, P=0.005$, and $P=0.001$, respectively) (Fig. 3). The visibility score was lower in the supine position than in the LLD position ( $P=0.018)$. However, there was no statistically significant difference between the visibility scores of the supine position and RPO position ( $\mathrm{P}=0.608$ ).

In 18 tumors, the visibility scores for the semi-erect position were equal to or lower than that of all the other positions. However, the visibility scores were lower in the RPO position than in the semi-erect position in two cases (score 2 vs. 3 and score 1 vs. 2, respectively), in which the tumors were located in the segment 8 dome.

\section{Technical Feasibility of Needle Insertion}

The technical feasibility of needle insertion is presented in Table 2 . According to the visibility score, the technical feasibility of needle

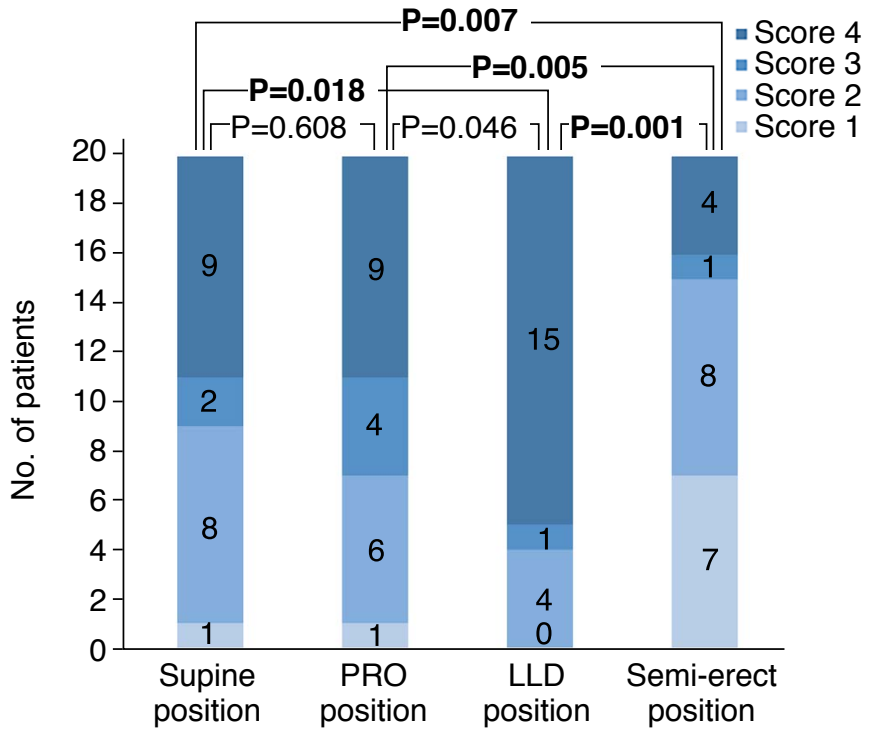

Fig. 2. Visibility scores in the four different positions.

The numbers in each column indicate the number of subjects with each visibility score. RPO, right posterior oblique; LLD, left lateral decubitus. Score 1, completely identifiable, highly confident in identifying the index tumor; score 2, partially (more than half of index tumor) identifiable, and confident in identifying the index tumor; score 3, partially (less than 1/2 of index tumor) identifiable; and score 4, definitely unidentifiable. The Wilcoxon signed rank test was used.

Table 2. Technical feasibility of needle insertion in the four body positions

\begin{tabular}{lcccc}
\hline & Supine & RPO & LLD & $\begin{array}{c}\text { Semi- } \\
\text { erect }\end{array}$ \\
\hline $\begin{array}{l}\text { Technical feasibility } \\
\text { of needle insertion, } \mathrm{n}(\%)^{\text {a) }}\end{array}$ & $9(45.0)$ & $7(35.0)$ & $5(25.0)$ & $15(75.0)$ \\
$\begin{array}{l}\text { P-value } \\
\text { R) }\end{array}$ & & & & \\
RPO & 0.687 & & & \\
LLD & 0.180 & 0.453 & & \\
Semi-erect & 0.031 & 0.021 & 0.001 &
\end{tabular}

RPO, right posterior oblique; LLD, left lateral decubitus.

${ }^{a}$ Needle insertion was considered to be technically feasible if the visibility score was equal to or lower than 2. ${ }^{\text {b) } M c N e m a r}$ test.

insertion was highest in the semi-erect position $(75 \%, 15 / 20)$, followed by the supine position $(45 \%, 9 / 20)$, the RPO position (35\%, $7 / 20)$, and the LLD position $(20 \%, 4 / 20)$. The technical feasibility of needle insertion was statistically higher in the semi-erect position than in the supine, RPO, and LLD positions ( $P=0.031, P=0.021$, and $P=0.001$, respectively). Technical feasibility was not statistically significantly different between the supine position and the RPO or LLD positions ( $P=0.687$ and $P=0.180$, respectively). Furthermore, the feasibility was not significantly different between the RPO and 


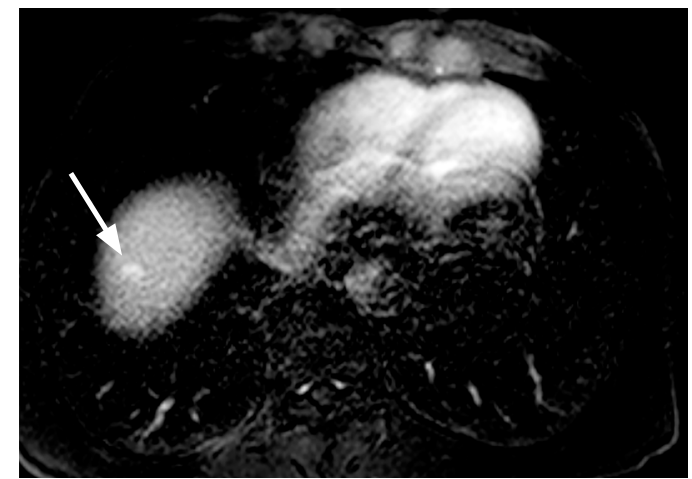

A

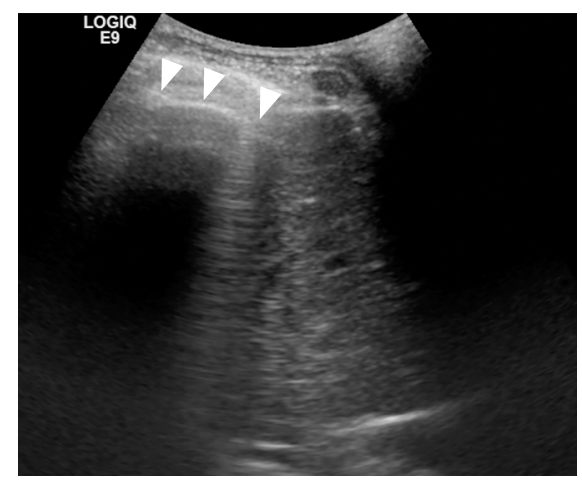

C

D

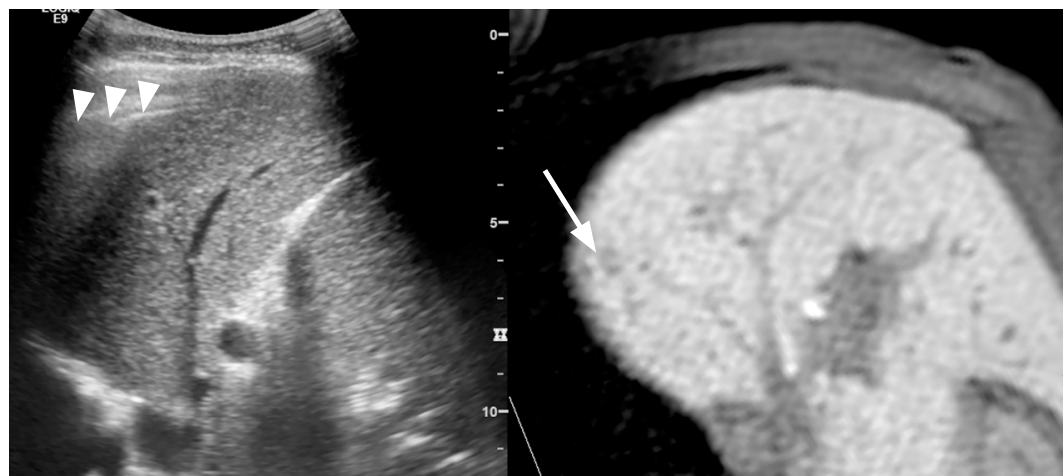

B

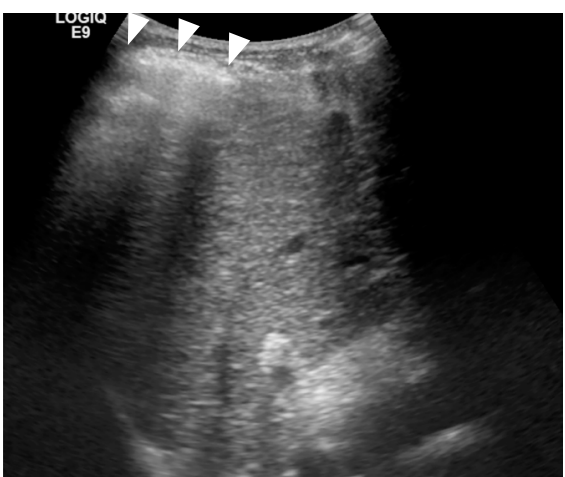

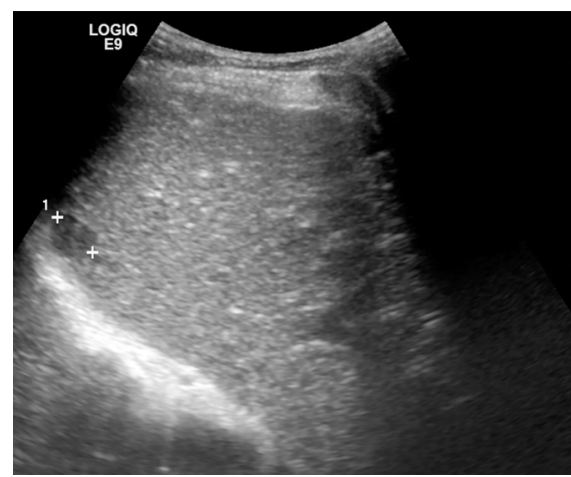

$\mathrm{E}$

Fig. 3. A 61-year-old man with liver cirrhosis due to hepatitis B viral infection.

A. Hepatic arterial phase magnetic resonance image shows a 1.1-cm subphrenic hepatocellular carcinoma (arrow). B. On planning ultrasonography with fusion imaging with the hepatobiliary phase image in supine position, the lesion (arrow) is obscured by the lung shadow (arrowheads) and is not able to be identified. Therefore, the visibility score was graded as 4 and needle insertion was considered infeasible. The lesion is not visualized due to the lung shadow (arrowheads) in both the right posterior oblique position (C) and the left lateral decubitus position (D). E. In the semi-erect position using a tilting table, the lesion is clearly visible as a hypoechoic nodule. The visibility score was graded as 1 and needle insertion was considered technically feasible.

LLD positions $(\mathrm{P}=0.453)$.

\section{Discussion}

In this study, we evaluated which body position was most useful for visualizing subphrenic HCCs and found that the semi-erect position had advantages over other positions, including the supine, RPO, and LLD positions, for detecting subphrenic HCCs. In addition, the technical feasibility of needle insertion was highest in the semi-erect position. Our results indicate that the semi-erect position can be utilized for better visualization of hepatic dome lesions during US examinations.

Traditionally, various positions of the patient's body, such as the supine, prone, erect, and LLD positions, have been attempted for better visualization of the hepatobiliary system during US examinations [4,12-14]. However, to our knowledge, the question of the most effective body position for visualization of the liver dome has not yet been evaluated thoroughly in a well-designed study. Among various body positions, the semi-erect position seems to be a valuable option for better visualization of the liver dome as the liver moves down with gravity in the semi-erect position. As shown in this study, the semi-erect position can be easily achieved by using an operating table that is capable of being tilted (Fig. 1). Therefore, the patient does not have to struggle to maintain this position and can simply lie down on the table with his or her hands raised and resting by the head. This semi-erect position on the operating table may have advantages over the Fowler position [15], in which the patient is seated in a semi-sitting position, because the intercostal space in the semi-erect position is wider than that in the semi-sitting position as body posture remains straight, not bent, in the semi-erect position. This may enable a more uninterrupted view of intercostal US scans through a wider intercostal space.

One concern is that tables with the capability of being tilted are more expensive than the conventional tables used for routine 
US examinations. Nevertheless, these tables would seem to be a worthwhile investment if they are used for both routine abdominal US examinations and interventional procedures for focal hepatic lesions. It is postulated that the US examination time may be reduced if the semi-erect position is used for routine abdominal US, as the sonographic window of the liver would be enhanced and the need for frequent position changes during the US examination may be eliminated. This assumption is partially supported by the fact that subphrenic HCCS are difficult to localize with US even when the radiologists are aware of the size and location of the tumor based on pre-acquired CT or MR images due to the poor sonographic window $[5,6,16]$. Because the adequacy of liver visualization may affect the sensitivity of US detection of a focal lesion, the US Liver Imaging Reporting and Data System for screening and surveillance of HCC recently proposed three categories to represent visualization scores: A, minimal limitations; B, moderate limitations; and C, severe limitations [4]. It is likely that the number of lesions classified as category $\mathrm{C}$ would be reduced by using the semi-erect position and that many patients would benefit from using this position when being examined with US for HCC surveillance. A well-designed prospective trial is warranted to verify this assumption.

The semi-erect position would be also useful during US-guided local ablation therapy for subphrenic tumors, particularly in segment 7. Although artificial ascites is frequently used to protect the adjacent diaphragm from thermal damage and to obtain a better sonographic window when treating hepatic dome lesions $[17,18]$, subphrenic tumors in segment 7 cannot be separated from the diaphragm due to the bare area of the liver in relation to the diaphragm. Therefore, artificial fluid is mainly used to enhance the sonographic window for tumors in segment 7. For this particular situation, artificial pleural effusion may be preferred over artificial ascites if the procedure is performed in the semi-erect position. This is because artificial ascites may not work well in the semierect position, as the infused fluid would move down to the pelvic cavity by gravity. However, if pleural effusion is present with a subphrenic tumor in segment 7, using the semi-erect position would be a valuable strategy, as a small amount of pleural effusion would accumulate in the basal thoracic cavity [15]. This implies that with the use of pleural effusion, we would not have to aspirate the artificial fluid after local ablation therapy to relieve the patient's discomfort caused by artificial effusion. Artificial pleural effusion has been reported to be advantageous by previous studies in which RFA was successfully performed after introducing artificial pleural effusion for subphrenic tumors in the semi-sitting position $[19,20]$.

Tumors in the liver dome that are not too close to the diaphragm, and therefore do not require artificial ascites for US-guided ablation procedures, would also benefit from the semi-erect position. When the liver moves down by gravity, the index tumor also moves downward. This means that the RFA needle trajectory for a hepatic dome lesion does not have to be at a steep angle when compared to the supine position, in which the liver and the index tumor are located at a higher level, within the rib cage. Consequently, straight RF electrodes do not have to move back and forth as frequently along with the patient's breathing motion, resulting in an ablation zone with a more favorable shape, close to a sphere, in the semierect position. To answer this assumption, further studies regarding RFA for dome HCCs with different positions of the patient's body are warranted.

This study has several limitations. First, the visibility score was graded by one of three radiologists. Therefore, this study may be limited by possible inter-observer variability in the visibility score assessment, even though the all three radiologists had some experience in US-guided RFA of HCCS. However, given that US examinations are done in real time, the assessment of inter-observer agreement was not practical in this study. Second, the value of the LPO position is unknown because it was not included for comparison in this study; the reason for this is that we postulated that the difference between the LLD and LPO positions for the evaluation of liver dome would not be very large. Third, fusion imaging was applied to estimate the tumor location only for the supine position, which may have affected the results of the US examinations with different body positions. However, applying fusion imaging for all the other positions was not practical because time is needed to fuse real-time US and CT/MR images. In addition, some changes could have taken place in the size and shape of liver in positions other than the supine position, as CT or MR images are routinely obtained in the supine position. Lastly, the direct advantage of the semi-erect position for percutaneous RFA of subphrenic HCCs could not be assessed in our study as most RFA procedures were performed in the supine position after introducing artificial ascites. Nevertheless, we believe that the semi-erect position would be useful for interventional procedures such as percutaneous biopsy, as the sonographic window for hepatic dome lesions is easily enhanced by simply changing the body position.

In conclusion, the semi-erect position was more useful for visualizing subphrenic HCCs during US examinations than the supine, RPO, and LLD positions.

ORCID: Seong Eun Ko: https://orcid.org/0000-0003-0007-6569; Min Woo Lee: https:// orcid.org/0000-0001-9048-9011; Hyo Keun Lim: https://orcid.org/0000-0003-32697503; Ji Hye Min: https://orcid.org/0000-0002-8496-6771; Dong Ik Cha: https:// orcid.org/0000-0003-3271-6532; Tae Wook Kang: https://orcid.org/0000-00020725-8317; Kyoung Doo Song: https://orcid.org/0000-0002-2767-3622; Min Ju Kim: https://orcid.org/0000-0001-9866-3790; Hyunchul Rhim: https://orcid.org/0000-00029737-0248 


\section{Author Contributions}

Conceptualization: Lee MW, Lim HK. Data acquisition: Lee MW, Lim HK, Rhim H. Data analysis or interpretation: Lee MW, Ko SE, Min JH, Cha DI, Kang TW, Song KD. Drafting of the manuscript: Ko SE, Lee MW, Kim MJ. Critical revision of the manuscript: Lee MW, Ko SE. Approval of the fnal version of the manuscript: all authors.

\section{Conflict of Interest}

No potential conflict of interest relevant to this article was reported.

\section{Acknowledgments}

This study received research funding from the Korean Liver Cancer Study Group.

\section{References}

1. European Association for the Study of the Liver. Corrigendum to "EASL Clinical practice guidelines: management of hepatocellular carcinoma" [J Hepatol 69 (2018) 182-236]. J Hepatol 2019;70:817.

2. Marrero JA, Kulik LM, Sirlin CB, Zhu AX, Finn RS, Abecassis MM, et al. Diagnosis, staging, and management of hepatocellular carcinoma: 2018 practice guidance by the American Association for the Study of Liver Diseases. Hepatology 2018;68:723-750.

3. Villanueva A, Minguez B, Forner A, Reig M, Llovet JM. Hepatocellular carcinoma: novel molecular approaches for diagnosis, prognosis, and therapy. Annu Rev Med 2010;61:317328.

4. Morgan TA, Maturen KE, Dahiya N, Sun MR, Kamaya A, American College of Radiology Ultrasound Liver Imaging and Reporting Data System (US LI-RADS) Working Group, et al. US LI-RADS: ultrasound liver imaging reporting and data system for screening and surveillance of hepatocellular carcinoma. Abdom Radiol (NY) 2018;43:41-55

5. Kim PN, Choi D, Rhim H, Rha SE, Hong HP, Lee J, et al. Planning ultrasound for percutaneous radiofrequency ablation to treat small $(\leq 3 \mathrm{~cm})$ hepatocellular carcinomas detected on computed tomography or magnetic resonance imaging: a multicenter prospective study to assess factors affecting ultrasound visibility. J Vasc Interv Radiol 2012;23:627-634.

6. Lee MW, Kim YJ, Park HS, Yu NC, Jung SI, Ko SY, et al. Targeted sonography for small hepatocellular carcinoma discovered by $\mathrm{CT}$ or MRI: factors affecting sonographic detection. AJR Am J Roentgenol 2010;194:W396-W400.

7. Lencioni R, Piscaglia F, Bolondi L. Contrast-enhanced ultrasound in the diagnosis of hepatocellular carcinoma. J Hepatol 2008;48:848857.

8. Park JH, Park MS, Lee SJ, Jeong WK, Lee JY, Park MJ, et al. Contrastenhanced US with perfluorobutane for hepatocellular carcinoma surveillance: a multicenter diagnostic trial (SCAN). Radiology 2019;292:638-646.

9. Bruix J, Sherman M; American Association for the Study of Liver Diseases. Management of hepatocellular carcinoma: an update. Hepatology 2011;53:1020-1022.

10. Lee MW, Rhim H, Cha DI, Kim YJ, Lim HK. Planning US for percutaneous radiofrequency ablation of small hepatocellular carcinomas $(1-3 \mathrm{~cm})$ : value of fusion imaging with conventional US and CT/MR images. J Vasc Interv Radiol 2013;24:958-965.

11. Song KD, Lee MW, Rhim H, Kang TW, Cha DI, Sinn DH, et al. Percutaneous US/MRI fusion-guided radiofrequency ablation for recurrent subcentimeter hepatocellular carcinoma: technical feasibility and therapeutic outcomes. Radiology 2018;288:878886.

12. Conrad MR, Leonard J, Landay MJ. Left lateral decubitus sonography of gallstones in the contracted gallbladder. AJR Am J Roentgenol 1980;134:141-144.

13. Hough DM, Glazebrook KN, Paulson EK, Bowie JD, Foster WL. Value of prone positioning in the ultrasonographic diagnosis of gallstones: prospective study. J Ultrasound Med 2000;19:633-638.

14. Dietrich CF, Serra C, Jedrzejczyk M. Ultrasound of the liver. In: Dietrich CF, ed. EFSUMB course book. London: European Federation of Societies for Ultrasound in Medicine and Biology, 2010;56-66.

15. Minami Y, Kudo M, Kawasaki T, Chung H, Ogawa C, Shiozaki $\mathrm{H}$. Percutaneous radiofrequency ablation guided by contrastenhanced harmonic sonography with artificial pleural effusion for hepatocellular carcinoma in the hepatic dome. AJR Am J Roentgenol 2004;182:1224-1226.

16. Kim JE, Kim YS, Rhim H, Lim HK, Lee MW, Choi D, et al. Outcomes of patients with hepatocellular carcinoma referred for percutaneous radiofrequency ablation at a tertiary center: analysis focused on the feasibility with the use of ultrasonography guidance. Eur J Radiol 2011;79:e80-e84.

17. Song I, Rhim H, Lim HK, Kim YS, Choi D. Percutaneous radiofrequency ablation of hepatocellular carcinoma abutting the diaphragm and gastrointestinal tracts with the use of artificial ascites: safety and technical efficacy in 143 patients. Eur Radiol 2009;19:2630-2640.

18. Rhim H, Lim HK, Kim YS, Choi D. Percutaneous radiofrequency ablation with artificial ascites for hepatocellular carcinoma in the hepatic dome: initial experience. AJR Am J Roentgenol 2008;190:91-98.

19. Koda M, Ueki M, Maeda Y, Mimura K, Okamoto K, Matsunaga Y, et al. Percutaneous sonographically guided radiofrequency ablation with artificial pleural effusion for hepatocellular carcinoma located under the diaphragm. AJR Am J Roentgenol 2004;183:583-588.

20. Uehara T, Hirooka M, Ishida K, Hiraoka A, Kumagi T, Kisaka Y, et al. Percutaneous ultrasound-guided radiofrequency ablation of hepatocellular carcinoma with artificially induced pleural effusion and ascites. J Gastroenterol 2007;42:306-311. 